\title{
Microcefalia: consideraciones para el abordaje diagnóstico
}

\section{Microcephaly: diagnostic considerations}

Gilda Garza-Mayén, ${ }^{1}$ Moisés Fiesco-Roa, ${ }^{2}$ Sara Frías, ${ }^{3}$ Benilde García-de Teresa ${ }^{4}$

\section{ANTECEDENTES}

La microcefalia, o disminución del tamaño del perímetro cefálico, es un signo reconocible en diversas entidades clínicas que cursan con alteraciones en el crecimiento craneal o del sistema nervioso central. Su definición en la bibliografía no es uniforme. Los CDC (Centers for Diseases Control and Prevention) y la American Academy of Pediatrics la definen como un perímetro cefálico menor a -2 desviaciones estándar para la media poblacional o por debajo del percentil 3 para la edad y sexo. ${ }^{1}$ A su vez, la Asociación Española de Pediatría la considera cuando el perímetro cefálico es inferior a -3 desviaciones estándar, ${ }^{2}$ mientras que la American Academy of Neurology utiliza este mismo criterio para determinar "microcefalia severa" en pacientes con perímetro cefálico inferior a -3 desviaciones estándar. En estos pacientes se ha observado, antes del nacimiento, mayor retraso en el neurodesarrollo y más alteraciones cerebrales estructurales. ${ }^{3,4}$. Desde luego que es posible detectarla en el periodo neonatal. La Sociedad de Medicina Materno-Fetal la define como una circunferencia cefálica fetal menor a -3 desviaciones estándar para la edad gestacional. La detección puede hacerse mediante ultrasonido estructural entre las 18 a 24 semanas de gestación, mientras que la resonancia magnética fetal permite evaluar alteraciones en el desarrollo cerebral. ${ }^{5}$ Para fines de este artículo se utilizará la definición clásica de microcefalia como un perímetro cefálico menor a -2 desviaciones estándar o por debajo del percentil 3.

Los términos "perímetro cefálico reducido" y "circunferencia occipitofrontal pequeña" pueden utilizarse como sinónimos de microcefalia, no así la palabra "microcráneo", que es preferible evitar porque se refiere solo a la estructura ósea. ${ }^{6,7}$ Las craneosinostosis pueden asociarse con disminución del perímetro cefálico pero no se consideran

\footnotetext{
${ }^{1}$ Residente de tercer año de Genética médica, Departamento de Genética Humana,

Instituto Nacional de Pediatría. orcid org/0000-0002-7895-7166

2 Programa de doctorado en Ciencias Médicas, Odontológicas y de la Salud, Universidad Nacional Autónoma de México, Laboratorio de Citogenética, Departamento de Genética Humana, Instituto Nacional de Pediatría. orcid. org/0000-0002-8123-5068

${ }^{3}$ Investigador en Ciencias Médicas F, Laboratorio de Citogenética, Departamento de Genética Humana, Instituto Nacional de Pediatría, Instituto de Investigaciones Biomédicas, Departamento de Medicina Genómica y Toxicología Ambiental, Universidad Nacional Autónoma de México. orcid.org/0000-0002-3097-6368

${ }^{4}$ Médico genetista, ayudante de investigador en Ciencias Médicas C, Laboratorio de Citogenética, Departamento de Genética Humana, Instituto Nacional de Pediatría.

orcid.org/0000-0002-9378-1007
}

Recibido: 19 de julio 2020

Aceptado: 21 de agosto 2020

Correspondencia

Benilde García-de Teresa

b.garciadeteresa@gmail.com

Este artículo debe citarse como Garza-Mayén G, Fiesco-Roa M, Frías S, García-de Teresa B. Microcefalia: consideraciones para el abordaje diagnóstico. Acta Pediatr Méx 2020; 41(5): 222-30. 
microcefalia como tal, porque la afección se localiza en el cráneo, por fusión de dos o más suturas. ${ }^{8}$ Los términos "microcefalia vera" o "microcefalia esencial" también deberían evitarse porque se utilizan con referencia predominante a las microcefalias primarias ${ }^{9,10} \mathrm{o}$ al subgrupo de microcefalias primarias hereditarias $(\mathrm{MCPH}$; MicroCephaly Primary Hereditary), que aunque son las más características cuando se describen de acuerdo con este parámetro, no son las únicas que conforman este grupo. ${ }^{2,9}$ Cuadro 1

\section{Epidemiología}

La frecuencia mundial de microcefalia al nacimiento varía de 1 a 56 casos por cada 10,000 recién nacidos. ${ }^{1,3}$ Esta frecuencia cambia según la región geográfica, presumiblemente por la variabilidad de causas de microcefalia. Por cada 10,000 recién nacidos se reportan 0.37 casos en México, 1.53 en Europa, en América del Sur 1 a 12 y en Estados Unidos 8.7. ${ }^{13} \mathrm{Al}$ gunos estudios reportan predominio en el sexo masculino $(59 \%){ }^{4}$

En 2015, la pandemia del virus Zika, en más de 80 países, reveló los efectos teratogénicos del virus en el sistema nervioso central y el aumento en la incidencia de microcefalia. En México se registraron $1 \%$ de los casos de Zika de América y la incidencia de microcefalia pasó de 0.37 antes del brote a $1.15,{ }^{11}$ lo que puso de manifiesto el impacto teratogénico del virus. Los estados con mayor cantidad son: Guerrero, Chiapas, Colima, Oaxaca, Veracruz y Tabasco en los que hay poblaciones ubicadas en altitudes inferiores a los 2200 m. ${ }^{11,14,15}$

\section{Etiología y clasificación}

Si bien muchos casos de microcefalia son de causa desconocida, puede ser consecuencia de un estímulo externo: infecciones, desnutrición y exposición a sustancias, o bien puede ser parte del fenotipo de una gran variedad de entidades genéticas. Hasta junio de 2020, en la base de datos OMIM (siglas en inglés de Online Mendelian Inheritance in Man) se habían reportado 996 entidades mendelianas que cursan con microcefalia. ${ }^{16}$

Una clasificación completa y adecuada facilita el establecimiento del diagnóstico del paciente, por lo que, de acuerdo con revisiones previas, ${ }^{2-4,17}$ consideramos clasificar la microcefalia en 5 categorías complementarias y no excluyentes.

\section{Cuadro 1}

El perímetro cefálico al nacimiento es un punto de referencia fundamental para clasificar la microcefalia de acuerdo con el tiempo de aparición y la evolución, por lo que es un dato que no debe faltar en la historia clínica. En México, el certificado de nacimiento no cuenta con un espacio designado para consignar el perímetro cefálico, lo que propicia dificultades para el abordaje diagnóstico.

\section{Evaluación y establecimiento del diagnóstico}

La evaluación inicial de un paciente con microcefalia debe ser metódica y requiere la adquisición de datos confiables y su correcta interpretación y clasificación.

\section{Medición}

El perímetro cefálico es una medición directa del tamaño craneal que, típicamente, refleja el tamaño del cerebro. Su medición correcta requiere el uso de una cinta métrica flexible para definir la circunferencia máxima de la cabeza pasando por tres puntos craneométricos: glabela, eurion y opistocráneo (Figura 1A). También es necesario obtener la medición en, al menos, dos ocasiones 
Cuadro 1. Clasificación de Microcefalia de Acuerdo con 5 Categorías para su Evaluación Inicial (continúa en la siguiente página)

\begin{tabular}{|c|c|c|c|}
\hline Categoría & Clasificación & Definición & Ejemplos \\
\hline \multirow[b]{2}{*}{ 1) Etiología } & Primaria & $\begin{array}{l}\text { Causa intrínseca del individuo, gene- } \\
\text { ralmente es congénita, acompañada } \\
\text { de trastornos del desarrollo cerebral } \\
\text { y de etiología genética. Frecuente- } \\
\text { mente está debajo de -3 DE. }\end{array}$ & $\begin{array}{l}\text { - MCPH (microcefalia primaria hereditaria), } \\
\text { antes conocidas como "microcefalia vera": } \\
\text { generalmente con patrón de herencia autosó- } \\
\text { mico recesivo, pero también se han reportado } \\
\text { autosómico dominante. } \\
\text { - Síndromes por defecto en la reparación del } \\
\text { ADN: anemia de Fanconi, Microcefalia pri- } \\
\text { mordial Osteodisplásica tipo 2, Xeroderma } \\
\text { pigmentoso, síndromes de Nijmegen, Seckel, } \\
\text { Cockayne. }\end{array}$ \\
\hline & Secundaria & $\begin{array}{l}\text { Causa adquirida, generalmente de } \\
\text { inicio postnatal (en los primeros años } \\
\text { de vida), acompañada de otros datos } \\
\text { de afección del SNC, de etiología } \\
\text { ambiental. }\end{array}$ & $\begin{array}{l}\text { - Errores innatos del metabolismo: defectos } \\
\text { de glicosilación, mitocondriopatías, enfer- } \\
\text { medades peroxisomales, aminoacidopatías } \\
\text { y acidemias orgánicas. } \\
\text { - Infecciones prenatales: TORCH, incluido } \\
\text { Zika. } \\
\text { - Infecciones postnatales: meningoencefalitis, } \\
\text { abscesos cerebrales. } \\
\text { - Teratógenos químicos: alcohol, cocaína, } \\
\text { tolueno, antiepilépticos (fenitoína, carbama- } \\
\text { zepina, valproato de sodio). } \\
\text { - Enfermedades vasculares: EVC (Evento Vas- } \\
\text { cular Cerebral) } \\
\text { - Enfermedades maternas: diabetes pregesta- } \\
\text { cional, hipotiroidismo, desnutrición, defi- } \\
\text { ciencia de folatos, anemia. }\end{array}$ \\
\hline \multirow[b]{2}{*}{$\begin{array}{l}\text { 2) Tiempo de } \\
\text { aparición }\end{array}$} & Congénita & $\begin{array}{l}\text { Presente al nacimiento, generalmen- } \\
\text { te se asocia a RCIU y talla baja de } \\
\text { inicio prenatal. }\end{array}$ & $\begin{array}{l}\text { - Síndrome de Cornelia de Lange: RCIU, retraso } \\
\text { del neurodesarrollo/DI, dismorfias faciales } \\
\text { (cejas pobladas, arqueadas y con sinofris, } \\
\text { pestañas largas, narinas antevertidas, filtrum } \\
\text { largo), hirsutismo, alteraciones de extremidades } \\
\text { (micromelia, contracturas codos), criptorquidia. } \\
\text { - Por TORCH, incluido Zika }\end{array}$ \\
\hline & Postnatal & $\begin{array}{l}\text { PC normal al nacimiento y posterior- } \\
\text { mente los valores quedan por debajo } \\
\text { de - } 2 \text { DE. }\end{array}$ & $\begin{array}{l}\text { - Síndrome de Angelman: retraso del neuro- } \\
\text { desarrollo/DI, alteraciones del movimiento } \\
\text { (ej., ataxia), risa inexplicable, conductas } \\
\text { esteriotipadas (Ej., aleteo de manos) } \\
\text { - Síndrome de Rett: regresión neurológica (en- } \\
\text { tre 1-4 años) con afectación motora, cognitiva } \\
\text { y del lenguaje, retraso del neurodesarrollo-DI, } \\
\text { movimientos esteriotipados (Ej., aplausos, } \\
\text { frotar las manos), rasgos autistas. } \\
\text { - Infecciones cerebrales del recién nacido y } \\
\text { el lactante }\end{array}$ \\
\hline \multirow[t]{2}{*}{ 3) Evolución } & Estática & $\begin{array}{l}\text { Permanece en un mismo estado } \\
\text { con el tiempo (Ej. dentro del mismo } \\
\text { rango de DE). }\end{array}$ & $\begin{array}{l}\text { - Síndrome de Bloom: RCIU, eritema telangiec- } \\
\text { tásico "en mariposa" en región malar que se } \\
\text { exacerba con la exposición solar, zonas de } \\
\text { hipo-hiperpigmentación. } \\
\text { - Consumo de fenitoína durante el embarazo. }\end{array}$ \\
\hline & Progresiva & $\begin{array}{l}\text { La gravedad de la microcefalia avan- } \\
\text { za o aumenta gradualmente. }\end{array}$ & $\begin{array}{l}\text { - Síndrome de Rett } \\
\text { - Fenilcetonuria no tratada: retraso del nuero- } \\
\text { desarrollo, crisis convulsivas, hipo-hiperexi- } \\
\text { tabilidad, hipopigmentación de piel y anexos. }\end{array}$ \\
\hline
\end{tabular}




\begin{tabular}{|c|c|c|c|}
\hline Categoría & Clasificación & Definición & Ejemplos \\
\hline \multirow{2}{*}{$\begin{array}{l}\text { 4) Proporción } \\
\text { respecto a la } \\
\text { talla }\end{array}$} & Proporcionada & $\begin{array}{l}\text { La microcefalia es proporcional a } \\
\text { la talla y, generalmente, también } \\
\text { al peso (ej. PC, peso y talla debajo } \\
\text { de }-2 \mathrm{DE} \text { ). }\end{array}$ & $\begin{array}{l}\text { - Síndrome por deleción 1p36: retraso del } \\
\text { neurodesarrollo/DI, alteraciones del com- } \\
\text { portamiento, dismorfias faciales (cejas rectas, } \\
\text { ojos hundidos, filtrum largo). } \\
\text { - Por desnutrición materna. }\end{array}$ \\
\hline & $\begin{array}{c}\text { No proporcionada } \\
\text { o Absoluta }\end{array}$ & $\begin{array}{l}\text { Unicamente PC está por debajo de } \\
-2 \mathrm{DE} \text {; la talla se encuentra en rango } \\
\text { normal para edad y sexo. }\end{array}$ & $\begin{array}{l}\text { - Síndrome de Seckel: RCIU, discapacidad } \\
\text { intelectual, convulsiones, dismorfias faciales } \\
\text { (frente inclinada, nariz prominente, microg- } \\
\text { natia). } \\
\text { - Parálisis cerebral asociada a daño cerebral } \\
\text { estático: infarto cerebral, asfixia perinatal }\end{array}$ \\
\hline \multirow[t]{2}{*}{ 5) Presentación } & Sindromática & $\begin{array}{l}\text { Cuando coexiste con otras altera- } \\
\text { ciones funcionales o del desarrollo } \\
\text { (malformaciones, deformaciones, } \\
\text { disrupciones o displasias). }\end{array}$ & $\begin{array}{l}\text { - Cromosómicas: Trisomía 13, } 18 \text { y } 21 \text {. } \\
\text { - Síndromes de microdeleción/duplicación: } \\
\text { Deleción 4p (Wolf-Hirschhorn), Deleción } \\
5 p \text { (Cri-du-chat), Deleción } 7 q 11 \text { (Williams). } \\
\text { - Monogénicas: Síndrome de Smith-Lemli- } \\
\text { Opitz, Síndrome de Kabuki. } \\
\text { - Desórdenes del espectro alcohol fetal: RCIU, } \\
\text { dismorfas faciales (fisuras palpebrales cortas, } \\
\text { labio superior delgado, filtrum plano), malfor- } \\
\text { maciones cardíacas y de SNC, convulsiones, } \\
\text { alteraciones del comportamiento. }\end{array}$ \\
\hline & No sindromática & $\begin{array}{l}\text { La microcefalia es aislada, no asocia- } \\
\text { da a otras alteraciones funcionales o } \\
\text { del desarrollo. }\end{array}$ & - Microcefalia familiar. \\
\hline
\end{tabular}

ADN: ácido desoxirribonucleico; DE: Desviación Estándar; PC: Perímetro Cefálico; DI: Discapacidad Intelectual; RCIU: Retraso del Crecimiento Intrauterino; SNC: Sistema Nervioso Central; TORCH: Toxoplasma, Otros, Rubeola, Citomegalovirus, Herpes. Nota: Los ejemplos no son exclusivos de las categorías y se pueden clasificar en más de una de estas. Las manifestaciones clínicas más características y prevalentes de los síndromes fueron tomadas de: 1) Lyons JK, et.al. Smith's Recognizable Patterns of Human Malformation, Elsevier $7^{\mathrm{a}}$ ed. 2013., 2) Smeets EEJ, et.al. Mol Syndromol 2011;2:113127. y 3) Denny L, et.al. Am Fam Physician 2017;96(8):515-522.

Ashwal S, 2009., Passemard S, 2013 Von der Hagen M, 2014., Harris SR, 2015., y Arroyo HA, 2018.

lo que asegura mayor certeza y precisión..$^{6,7}$ En el recién nacido se recomienda medir después de las primeras 24 horas de vida, posterior a que se resuelva el moldeamiento craneal propio del parto; sin embargo, en la mayoría de los centros hospitalarios esta medición se obtiene solo inmediatamente después del nacimiento. ${ }^{3}$

\section{Interpretación según la edad y sexo}

La interpretación de la medición del perímetro cefálico requiere la comparación del valor obtenido vs los parámetros poblacionales acordes con la edad y sexo. Cuando se conoce la edad gestacional, la Organización Mundial de la Salud recomienda los criterios de INTERGROWTH-21 para prematuros ${ }^{18} \mathrm{O}$, en su defecto, las gráficas de la OMS "Estándares del crecimiento infantil", que son las recomendadas para el seguimiento hasta los 5 años de edad, momento en que el crecimiento de la cabeza ya no es sustancial. ${ }^{6,19}$ Además de éstas, los CDC también tienen gráficas para población pediátrica. Aunque menos utilizadas, existen algunas para seguimiento hasta los 16 o, incluso, 21 años de edad. ${ }^{6,20}$ Las ideales son las gráficas para la población de 
origen. Ramos Galván publicó datos de población mexicana con los que pueden calcular las desviaciones estándar por edad y sexo a partir del mes hasta los 18 años(Figura 1B). ${ }^{21}$ Los datos de la talla y el peso son comparables con las tablas de crecimiento de los CDC, ${ }^{22}$ aunque esto no se ha validado para el perímetro cefálico.

En la actualidad se dispone de tablas y calculadoras en línea que facilitan la interpretación de la medición. Cuadro 2
Cuadro 2. Ligas de interés

Tablas de crecimiento

- OMS

- www.who.int/childgrowth/standards/hc_for_age/en

- CDC

- www.cdc.gov/growthcharts

- Ramos Galván

- https://pubmed.ncbi.nlm.nih.gov/1200734/

\section{Calculadoras}

- INTERGROWTH-21

- https://intergrowth21.tghn.org/standards-tools/

- Ped(z) Pediatric Calculator

- https://www.pedz.de/en/calculators.html

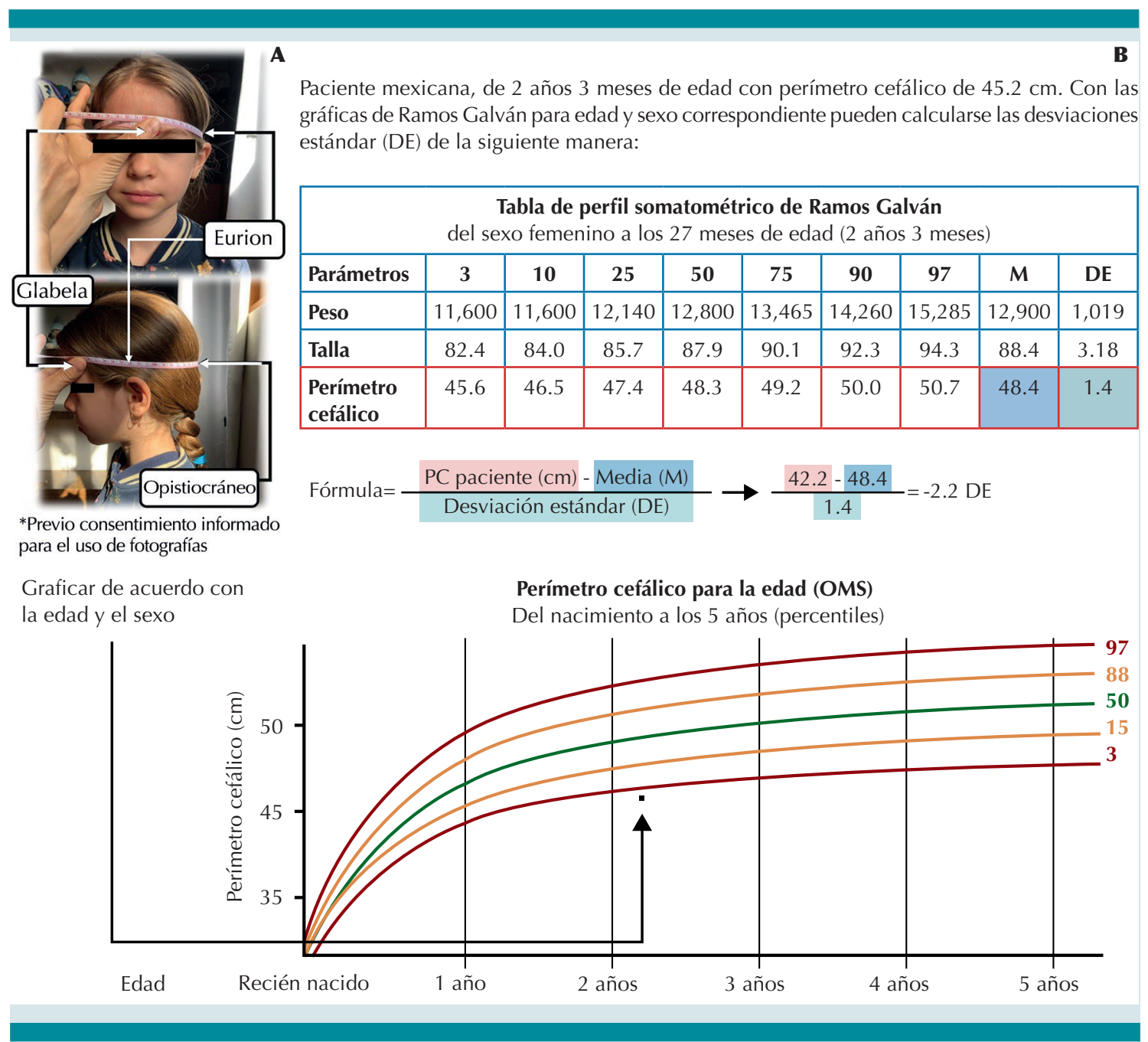

Figura 1. Medición e interpretación correcta del perímetro cefálico con cálculo de las desviaciones estándar de acuerdo con las tablas de Ramos Galván. 


\section{Integración diagnóstica}

Todos los pacientes con diagnóstico de microcefalia requieren: a) recolección y análisis de los antecedentes prenatales, gestacionales y familiares. Es importante medir y graficar el perímetro cefálico de ambos padres porque puede tratarse de un rasgo familiar; ${ }^{4}$ b) exploración física detallada; c) estudios complementarios: ${ }^{3,17}$

- Estudio de neuroimagen. De acuerdo con la sospecha diagnóstica puede ser de utilidad un ultrasonido transfontanelar, tomografía computada o resonancia magnética para identificar las alteraciones estructurales, que convendrá evalúe el neurólogo.

- Estudios genéticos. Puesto que suelen ser costosos y con diferente alcance diagnóstico y finalidad, dependiendo de la sospecha diagnóstica, siempre debe contarse con una evaluación previa por Genética y una consulta subsecuente para completar el asesoramiento genético.

- Otros. Biometría hemática y química sanguínea (anemia, infecciones, estados agudos, etc.). Fondo de ojo (coriorretinitis, puede sugerir TORCH). Tamiz neonatal (hipotiroidismo congénito, galactosemia clásica, fenilcetonuria, hiperplasia suprarrenal congénita por deficiencia de 21-hidroxilasa). Tamiz metabólico ampliado (errores innatos del metabolismo de molécula pequeña). Serología TORCH incluido Zika (considerar el periodo para tomar ese estudio y parearlo con la serología de la madre). Debe considerarse que todos los casos requieren un abordaje y manejo interdisciplinario en el que pueden intervenir las especialidades necesarias para individualizar cada caso según las características y necesidades de cada paciente.

\section{Diagnóstico}

El diagnóstico de microcefalia se establece cuando el perímetro cefálico del paciente es menor a -2 desviaciones estándar o por debajo del percentil 3, según la edad y el sexo. ${ }^{1}$ El diagnóstico etiológico se establece con base en los datos de la historia clínica, los antecedentes, las manifestaciones clínicas asociadas y los estudios de laboratorio y gabinete complementarios. El estudio del paciente con microcefalia favorece la posibilidad de establecer el diagnóstico más temprano o, en su defecto, disponer de información valiosa para su atención en instituciones de tercer nivel. Figura 2

\section{Asociaciones}

La microcefalia es un hallazgo que suele ir acompañado de otras manifestaciones, casi siempre neurológicas. El $65 \%$ de los casos se asocia con retraso en el neurodesarrollo y discapacidad intelectual; de 28.3 a $43 \%$ cursan con crisis convulsivas e incluso $76 \%$ de los pacientes evaluados con resonancia magnética tienen hallazgos en el sistema nervioso central (anomalías en la sustancia blanca, alteraciones estructurales) que pueden empeorar el pronóstico del neurodesarrollo. ${ }^{2,4,16}$ Otras alteraciones del desarrollo asociadas son: oftalmológicas (30\%), cardiopatías (14\%), esqueléticas (14\%) y genitourinarias (13\%). En muchos de estos casos, al final, se identifica una causa sindromática. , $16,23^{2}$

\section{¿Cuándo y a quién referir?}

Todos los pacientes con microcefalia detectada en cualquier momento de la infancia deben ser 


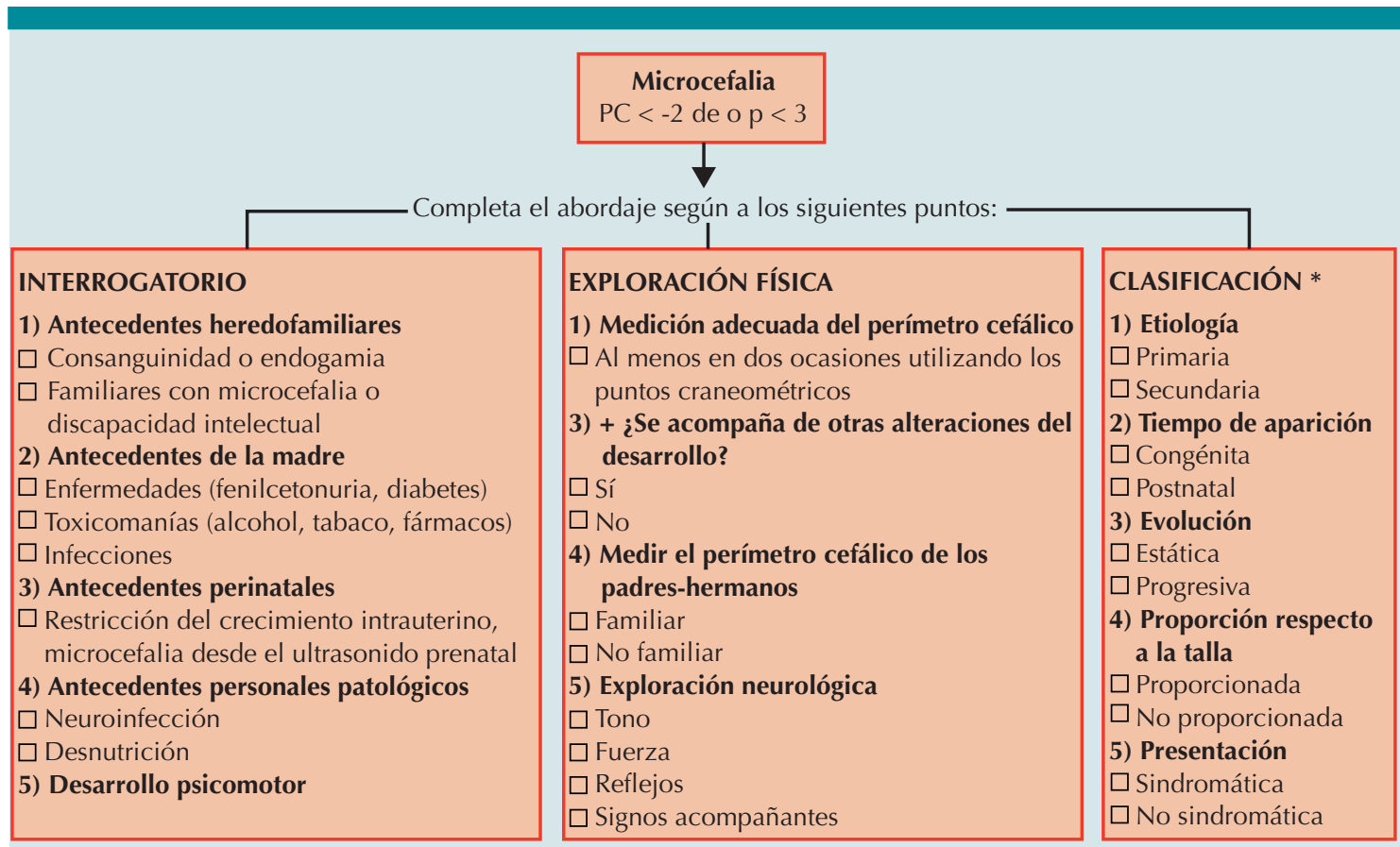

+ Las alteraciones del desarrollo pueden ser malforaciones, deformaciones, disrrupciones o displasias. * Las definiciones, ejemplos de la clasificación se encuentran en el Cuadro 1.

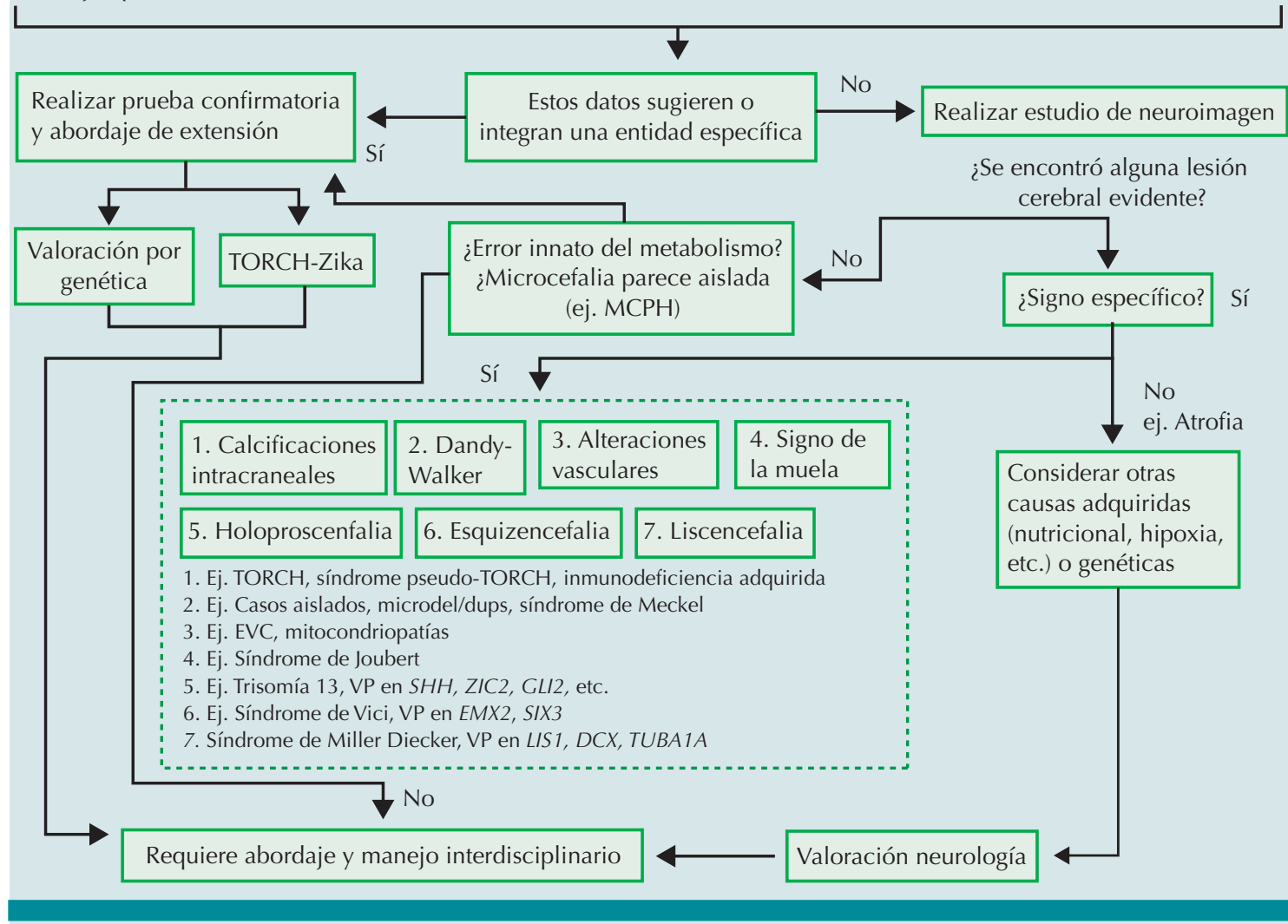

Figura 2. Abordaje inicial del paciente con microcefalia. 
atendidos en centros de tercer nivel de atención, sobre todo si:

- Tienen antecedente de consanguinidad, hermanos con microcefalia o factores de riesgo prenatal (Ej., teratógenos).

- La detección es en la etapa prenatal o al nacimiento. ${ }^{24,25}$

- Se acompaña de retraso global del neurodesarrollo, discapacidad intelectual, regresión u otras alteraciones neurológicas o del desarrollo. ${ }^{4}$

- La positividad, en algún estudio de laboratorio o gabinete, que sugiera un trastorno que pueda requerir tratamiento en etapas tempranas (errores innatos del metabolismo, infecciones y otras). ${ }^{14}$

Dependiendo de los hallazgos deberá valorarse referir a los servicios de Neurología, Infectología o Genética para disponer del diagnóstico de confirmación oportuno, valorar las opciones terapéuticas, de seguimiento y el asesoramiento en relación con el pronóstico o los riesgos de recurrencia, en caso de causas genéticas.

\section{Tratamiento y seguimiento}

A pesar de que para la mayoría de los casos no hay un tratamiento curativo, el diagnóstico temprano facilita la intervención y seguimiento efectivos, que se reflejan en el pronóstico del paciente. La microcefalia secundaria, o adquirida, puede prevenirse en algunos escenarios con dieta y complementos alimenticios que se inician desde el nacimiento; por ejemplo, los pacientes con fenilcetonuria u otros errores innatos del metabolismo. ${ }^{26}$ En casos de desnutrición de la madre se ha visto que una recuperación nutricional postnatal puede mejorar el perímetro cefálico. ${ }^{18}$ En la bibliografía hay evidencia que apoya que los niños con microcefalia y retraso en el neurodesarrollo deben ingresar a programas de rehabilitación física, del lenguaje y ocupacional de manera temprana, pues esas intervenciones repercuten postivamente en el desarrollo. Así mismo, el tratamiento farmacológico para el control de las crisis convulsivas es indispensable y disminuye las secuelas neurológicas. ${ }^{2,24}$

\section{Puntos clave}

- La medición del perímetro cefálico es una herramienta rápida, simple y no invasiva que permite establecer el diagnóstico de microcefalia.

- La medición del perímetro cefálico debe hacerse al nacimiento y periódicamente, al menos, hasta los 5 años de edad. ${ }^{4}$ La medición de forma seriada (inclusive después de los 5 años) puede ser de utilidad para el diagnóstico de pacientes con microcefalia postnatal, en casos de microcefalia progresiva o para identificar tempranamente complicaciones. ${ }^{2}$

- Con independencia del motivo de consulta, y como parte de la exploración integral en todos los pacientes debe medirse el perímetro cefálico, sobre todo en: 1) los que de forma subjetiva se observe una "cabeza pequeña"; 2) en quienes tengan factores de riesgo de daño neurológico; y 3) cuando haya otras manifestaciones acompañantes.

- La interpretación y análisis correctos del perímetro cefálico, así como su clasificación, son recursos valiosos para poder establecer el diagnóstico. 


\section{Agradecimientos}

Agradecemos a la Dra. Esther Lieberman la lectura crítica de este manuscrito y sus valiosos comentarios.

\section{REFERENCIAS}

1. Kalmin MM, et al. Misclassification in defining and diagnosing microcephaly. Paediatr Perinat Epidemiol. 2019; 33 (4): 286-90. doi:10.1111/ppe.12561

2. Harris SR. Measuring head circumference: Update on infant microcephaly. Can Fam Physician. 2015; 61 (8): 680-84.

3. Ashwal S, et al. Practice Parameter: Evaluation of the child with microcephaly (and evidence-based review): Report of the Quality Standards Subcommittee of the American Academy of Neurology and the Practice Committee of the Child Neurology Society. Neurology. 2009; 73 (13): 887-97. doi:10.1212/WNL.0b013e3181d5e057

4. Von der Hagen $M$, et al. Diagnostic approach to microcephaly in childhood: A two-center study and review of the literature. Dev Med Child Neurol. 2014; 56 (8): 732-41. doi:10.1111/dmcn.12425

5. SMFM Publications Committee. Ultrasound screening for fetal microcephaly following Zika virus exposure. In: American Journal of Obstetrics and Gynecology. Vol 214. Elsevier Ltd; 2016:B2-B4. doi:10.1016/j.ajog.2016.02.043

6. Hall JG, et al. Chapter 6. Head Circumference (Occipitofrontal Circumference, OFC). In: Oxford University Press I, ed. Handbook of Physical Measurements. $2^{\text {nd }}$ ed. New York, 2007; 72-83.

7. Allanson JE, et al. Elements of morphology: Standard terminology for the head and face. Am J Med Genet Part A. 2009; 149 (1): 6-28. doi:10.1002/ajmg.a.32612

8. Wang JC, Nagy L, Demke JC. Syndromic Craniosynostosis. Facial Plast Surg Clin North Am. 2016; 24 (4): 531-43. doi:10.1016/j.fsc.2016.06.008

9. Jayaraman D, et al. The Genetics of Primary Microcephaly. Annu Rev Genom Hum Genet. 2018; 19: 14.1-14.24. doi:10.1146/annurev-genom-083117-021441

10. Martí Herrero $\mathrm{M}$, et al. Macro y microcefalia. trastornos del crecimiento craneal. In: Protocolos Diagnóstico Terapeúticos de la AEP: Neurología Pediátrica. 2008; 185-193.

11. Hernández-Ávila JE, et al. Zika virus infection estimates, Mexico. Bull World Health Organ. 2018; 96 (5): 306-13. doi:10.2471/BLT.17.201004.
12. Morris JK, et al. Prevalence of microcephaly in Europe: Population based study. BMJ. 2016; 354: 1-6. doi:10.1136/ bmj.i4721

13. Cragan JD, et al. Population-based microcephaly surveillance in the United States, 2009 to 2013: An analysis of potential sources of variation. Birth Defects Res Part A - Clin Mol Teratol. 2016; 106 (11): 972-82. doi:10.1002/bdra.23587

14. Devakumar D, et al. Infectious causes of microcephaly: epidemiology, pathogenesis, diagnosis, and management. Lancet Infect Dis. 2018; 18 (1): e1-e13. doi:10.1016/S14733099(17)30398-5

15. Dirección General de Epidemiológia. Casos por entidad federativa: Displasias y neoplasias. In: Boletín Epidemiológico. Sistema Nacional de Vigilancia Epidemiológica. Vol 34. 2017:60-61.

16. OMIM. Online Mendelian Inheritance in Man Compendium. Entries with: Genemap for "microcephaly." Johns Hopkins University. https://omim.org/search?index=ent ry\&search=microcephaly\&start=1\&limit=10\&retrieve=ge neMap\&genemap_exists=true.

17. Arroyo HA, et al. Microcefalia. Medicina: Buenos Aires, 2018; 78: 94-100.

18. Villar J, et al. International standards for newborn weight, length, and head circumference by gestational age and sex: The Newborn Cross-Sectional Study of the INTERGROWTH-21st Project. Lancet. 2014; 384 (9946): 857-68. doi:10.1016/s0140-6736(14)60932-6

19. Child Growth Standards. Head Circumference-for-Age. World Health Organization. www.who.int/childgrowth/ standards/hc_for_age/en/. Published 2014.

20. Rollins JD, et al. United States Head Circumference Growth Reference Charts: Birth to 21 Years. J Pediatr. 2010;156(6):907-913.e2. doi:10.1016/j.jpeds.2010.01.009

21. Ramos-Galvan R. Pediatric somatometry. Semilongitudina study of children in Mexico City. Arch Invest Med. 1975; 6 (Suppl 1): 83-396.

22. Del-Rio-Navarro BE, et al. Mexican anthropometric percentiles for ages 10-18. Eur J Clin Nutr. 2007; 61 (8): 963-75. doi:10.1038/sj.ejcn.1602612

23. Vargas JE, et al. Congenital microcephaly: Phenotypic features in a consecutive sample of newborn infants. J Pediatr. 2001; 139 (2): 210-14. doi:10.1067/mpd.2001.115314

24. Nawathe A, et al. Fetal microcephaly. BMJ. 2018; 361: 1-5. doi:10.1136/bmj.k2232

25. 25. Marino S, et al. Congenital genetic microcephaly: Clinical diagnostic approach. J Pediatr Neurol. 2020; 18 (3): 131-34. doi:10.1055/s-0039-1692970

26. Passemard S, et al. Microcephaly. Handb Clin Neurol. 2013; 111: 129-41. doi:10.1016/B978-0-444-52891-9.00013-0 